\title{
Long Time Behavior for the Focusing Nonlinear Schroedinger Equation with Real Spectral Singularities
}

\section{Spyridon Kamvissis}

Universitć Paris-XIII, and Ecole Normale Supérieure, Cachan, France

Received: 5 Decembe1 1994/Accepted: 11 January 1996

\begin{abstract}
We consider the effect of real spectral singularities on the long time behavior of the solutions of the focusing Nonlinear Schroedinger equation We find that for each spectral singularity $\iota^{\prime} \in \mathbb{R}$, such an effect is limited to the region of the $(x, t)$-plane in which $i^{\prime}$ is close to the point of stationary phase $\lambda_{0}=\frac{-x}{4 t}$ (the phase here being defined in a standard way by, say, the evolution of the Jost functions) In that region, the solution performs decaying oscillations of the same form as in the other regions, but with different parameters The order of decay is $O\left(\left(\frac{\log t}{t}\right)^{12}\right)$

We prove our result by using the Riemann-Hilbert factorization formulation of the inverse scattering problem We recover our asymptotics by transforming our problem to one which is equivalent for large time, and which can be interpreted as the one corresponding to the genus 0 algebro-geometric solution of the equation
\end{abstract}

\section{Introduction}

We consider the nonlinear Schroedinger equation (focusing case)

$$
i q_{t}+q_{\mathrm{rr}}+2 q|q|^{2}=0
$$

under initial data

$$
q(x, 0)=q_{0}(x),
$$

belonging in the Schwartz class

As is well known (see [NMPZ], [FT]), the problem (1 1 )-(1 12 ) can be integrated through the method of inverse scattering. We will present here some of the results we will need without proof

The associated linear system is

$$
\psi_{\iota}=\left(\begin{array}{cc}
i \lambda & i q(x) \\
i \bar{q}(x) & -i \lambda^{\prime}
\end{array}\right) \psi
$$


where the bar denotes conjugation Jost functions $\psi_{1}, \psi_{2}, \phi_{1}, \phi_{2}$ are defined on the real line as (column vector) solutions of (1 3 ) satisfying the asymptotic conditions

$$
\begin{aligned}
& \psi_{1}(x, i) \sim\left(\begin{array}{c}
e^{i \lambda x} \\
0
\end{array}\right), \quad \text { as } x \rightarrow+\infty, \\
& \psi_{2}(x, \lambda) \sim\left(\begin{array}{c}
0 \\
e^{-i \lambda x}
\end{array}\right), \quad \text { as } x \rightarrow+\infty, \\
& \phi_{1}(x, \lambda) \sim\left(\begin{array}{c}
e^{i / x} \\
0
\end{array}\right), \quad \text { as } x \rightarrow-\infty, \\
& \phi_{2}(x, \lambda) \sim\left(\begin{array}{c}
0 \\
e^{-i \lambda \lambda}
\end{array}\right), \quad \text { as } x \rightarrow-\infty .
\end{aligned}
$$

Furthermore, $\psi_{1}$ and $\phi_{2}$ can be meromorphically extended to the upper half-plane, while $\psi_{2}$ and $\phi_{1}$ can be meromorphically extended to the lower half-plane Indeed, one has

$$
\begin{array}{ll}
\left(\psi_{1}(x, i) e^{-i \lambda}\right. & \left.\phi_{2}(x, i) e^{i \lambda}\right) \rightarrow I, \quad \text { as } i \rightarrow \infty, \quad \operatorname{Im} i \geqq 0, \\
\left(\phi_{1}(x, i) e^{-i \lambda}\right. & \left.\psi_{2}(x, i) e^{i \lambda x}\right) \rightarrow I, \quad \text { as } i \rightarrow \infty, \quad \operatorname{Im} i<0
\end{array}
$$

$I$ is here the identity matrix

We point out the symmetry

$$
\bar{\psi}(x, \bar{i})=\left(\begin{array}{cc}
0 & 1 \\
-1 & 0
\end{array}\right) \psi(x, i),
$$

where $\psi=\left(\begin{array}{ll}\psi_{1} & \psi_{2}\end{array}\right)$, the same symmetry is satisfied by $\phi=\left(\begin{array}{ll}\phi_{1} & \phi_{2}\end{array}\right)$

On the real line, $\phi$ and $\psi$ are solutions of system (13) Hence there exist "scattering coefficients" $a(\hat{i}), b(\hat{\lambda})$ such that

$$
\begin{aligned}
& \phi_{2}(x, \hat{i})=a(\hat{i}) \psi_{2}(x, \hat{i})+b(\lambda) \psi_{1}(x, \hat{i}), \\
& \phi_{1}(x, \hat{i})=\bar{a}(\hat{i}) \psi_{1}(x, \hat{i})-\bar{b}(\hat{i}) \psi_{2}(x, \hat{i})
\end{aligned}
$$

Although $\bar{a}$ and $\bar{b}$ are a priori independent of $a$ and $b$, one can see from the symmetries above that they are after all their conjugates Furthermore one can show

$$
|a(\lambda)|^{2}+|b(\lambda)|^{2}=1
$$

The time evolution of the scattering coefficients is given by

$$
\begin{aligned}
& a(\lambda, t)=a(\lambda, 0), \\
& b(\lambda, t)=b(\lambda, 0) e^{4 i \lambda^{2} t}
\end{aligned}
$$

It turns out that $a(\lambda)$ can be analytically extended to the upper half-plane, while $\bar{a}(i)$ can be analytically extended to a function $a^{*}\left(i_{i}\right)=\bar{a}(\bar{\lambda})$ in the lower half-plane 
In general, this is not also true for $b$. Generically, $a$ has only a finite number of zeros in the upper half-plane and no zero at all on the real line (cf [BC]) However, there are cases where this is not true One can have, for example, an infinity of non-real zeros with a limit point on the real line (see [Z] for an example)

It is well-known that in the generic case, non-real zeros of $a$ correspond to solitons for long times (see [FT] for example) Our goal is to study the effect of real zeros of a We will only consider the case of finitely many zeros (real or not) This is indeed the case if we restrict ourselves to initial data such that

$$
\int_{-\infty}^{\infty} e^{\eta|x|}\left|u_{0}(x)\right| d x<\infty, \quad \text { for some } \eta>0
$$

(cf. [F] for a proof) For simplicity we will state and prove our results in the case where no non-real zeros of $a$ are present and only one real simple zero exists However, it should eventually become clear that this is only a superficial constraint, and indeed we will indicate at the end of this work what happens in the more general case (of finitely many zeros)

An example of initial data producing exactly one real spectral singularity is the following ([CK]). Let $X>0$ and

$$
\begin{aligned}
u_{0}(x) & =\frac{\pi}{2 X}, \quad 0<x<X, \\
& =0, \quad \text { otherwise }
\end{aligned}
$$

Then $a(i)$ has exactly one (simple) zero at $i=0$ and $a^{\prime}(0)=\frac{-2 i x}{\pi}$

We define the $2 \times 2$-matrix-valued function $\Psi$ as follows Let

$$
\begin{array}{rlr}
\Psi & =\left(\begin{array}{ll}
\psi_{1} e^{-i \lambda} & \frac{\phi_{2} e^{i \lambda}}{a(\lambda)}
\end{array}\right), \quad \text { for } \operatorname{Im} \lambda>0, \\
& =\left(\begin{array}{ll}
\frac{\phi_{1} e^{-i \lambda}}{a^{*}(\lambda)} & \psi_{2} e^{i \lambda}
\end{array}\right), \quad \text { for } \operatorname{Im} i<0
\end{array}
$$

Letting $\Psi_{+}$and $\Psi_{-}$denote the limits of $\Psi$ on the real line from above and below respectively, we have (after a few calculations)

$$
\Psi_{+}(z)=\Psi_{-}(z)\left(\begin{array}{cc}
1 & r(i) e^{2 i \lambda+4 i^{2} t} \\
\bar{r}(\lambda) e^{-2 i \lambda_{-}-4 \iota^{2} t} & 1+|r(i)|^{2}
\end{array}\right), \quad \operatorname{Im} i=0,
$$

where $r(i)=\frac{b(\lambda, t=0)}{a(i, t=0)}$. Note that the jump matrix has determinant 1

Finally, one can prove that $a(\lambda)=1+O(1 / \lambda)$ as $\lambda \rightarrow \infty, \operatorname{Im} \lambda \geqq 0$ Hence

$$
\Psi(\hat{\lambda}=\infty)=I
$$

We thus end up with a Riemann-Hilbert factorization problem. $\Psi$ is a matrix function, analytic in the complement of the real line, satisfying the jump condition (1.12) and the asymptotic condition (113).

Note that in the generic case of finitely many zeros off the real line and no real zero, $\Psi$ is meromorphic and neither $\Psi_{+}$or $\Psi_{-}$nor the jump matrix have any singularities In the case we are interested in, however, both $\Psi_{ \pm}$and the jump matrix have singularities exactly at the zeros of $a(i)$. 
Conversely, the solution of the Riemann-Hilbert problem enables us to recover $q(x, t)$ Indeed (see e g [NMPZ])

$$
q(x, t)=-2 \lim _{j \rightarrow \infty} i \Psi_{12}
$$

Thus, the initial value problem $\left(\begin{array}{ll}1 & 1\end{array}\right)-\left(\begin{array}{ll}1 & 2\end{array}\right)$ is reduced to the above Riemann Hilbert on which we focus from now on

The interest of the problem treated in this paper is twofold On the first hand, as we will see later, the physical effect of the spectral singularity is a "collisionless shock" type phenomenon; we thus have an interesting connection with the theory of "dispersive shocks" for nonlinear wave equations (cf. also [DVZ], [AS]) On the other hand, ours is a first step towards the completion of the solution of the problem of long-time asymptotics of integrable equations in the case of Schwartz data, in the following sense although the related direct and inverse scattering problems are now completely solved (see [Z], [DZ2]) for the most general cases of Lax operators and even in the non-generic exceptional cases of data for which there are either infinitely many spectral singularities off the associated Riemann-Hilbert contour or (possibly infinitely many) spectral singularities on the contour, the longtime asymptotics problem is still far from having a complete solution, even in cases as simple as the NLS equation In fact, the present paper is the second of a series, in a recent article (see $[\mathrm{K}]$ ) we treat the infinite-soliton case, while a treatment of the case of infinitely many real (i e on the Riemann-Hilbert contour) spectral singularities for the NLS equation will appear soon

We now state our main result, to be proved in Sect 3 .

Theorem 1.1. Let $q$ be the solution of (1 1) with initial data in the Schwartz class, and such that a (simple) spectral singularity exists at $i=0$ and nowhere else Let $i_{0}=\frac{-1}{4 t}, \tau=t i_{0}^{2}$ and $K, M$ be given positive constants Then the leading order asymptotics of $q$, as $t \rightarrow \infty$, is as follow's

In region $I x<0, \hat{i}_{0}<-M$, q is given by formulae (2.5) and (2 6)

In region $I I x<0, \lambda_{0} \rightarrow 0, \tau \rightarrow \infty, q$ is given by formula (3 18)

In region $I I I \lambda_{0} \rightarrow 0, \tau<M, q$ is given by (3.19)

In region $I V \quad x>0, \lambda_{0} \rightarrow 0, \tau \rightarrow \infty, q$ is given by (3 18)

In region $V x>0, i_{0}>M, q$ is given by' (2 5)-(2 6)

The plan of the rest of the paper is as follows. In Sect.2, we solve the Riemann-Hilbert factorization problem in the case where the zeroes of $a$, are away from the stationary point $i_{0}=\frac{-r}{4 t}$ of the phase $\Theta=i x+2 i^{2} t$ Indeed, we show that no spectral singularity has any effect at all in the long time behavior of $q(x, t)$ In Sect 3, we consider the more interesting case where $\lambda_{0}-i^{\prime} \rightarrow 0$ with time, for some real singularity $\imath^{\prime}$ In Sect 4 , we discuss a generalization of our results

The problem of a real spectral singularity was first considered by Ablowitz and Segur In [AS] they study the collisionless shock phenomenon for the $\mathrm{KdV}$ equation with decaying initial data and they dedicate a small section to the focusing nonlinear Schroedinger equation, where they address the problem of a real spectral singularity by considering non-real ones, say $\kappa_{0}$, and taking the limit as $\operatorname{Im} \kappa_{0} \rightarrow 0$ Their treatment is heuristic and non-rigorous It is satisfactory however that their estimate for the decay of the solution on the "shock" front agrees with ours. On the other hand there are two discrepancies, concerning the phase and the coefficient of the 
decay term For the sake of the reader, we present the result of Ablowitz and Segur below

Result of $[A S]$ In the shock front region (corresponding to our regions II and IV), the solution of ( $\left.\begin{array}{ll}1 & 1\end{array}\right)-\left(\begin{array}{ll}1 & 2\end{array}\right)$ has the following asymptotic expression

$$
q(x, t)=t^{-12} R(x / t, t) e^{i t(t)(x t, t)}
$$

where

$$
\begin{gathered}
O \sim \frac{1}{4} \frac{x^{2}}{t^{2}}+2 \frac{\log t}{t} f^{2}\left(\frac{x}{t}\right)+O\left(\frac{1}{t}\right), \\
R \sim f\left(\frac{x}{t}\right)+4 f\left(\frac{x}{t}\right)\left(3\left[f^{\prime}\left(\frac{x}{t}\right)\right]^{2}+f\left(\frac{x}{t}\right) f^{\prime \prime}\left(\frac{x}{t}\right)\right) \frac{\log t}{t}, \\
f^{2}(-4 k) \sim \frac{1}{4 \pi}(\log t-\log \log t-2 \log m), \quad \text { where } k=i^{\prime}+m\left(\frac{\log t}{t}\right)^{12}
\end{gathered}
$$

In particular (cf $(410)$ of $[\mathrm{AS}])$

$$
|q| \sim \frac{1}{2 \pi^{1 / 2}}\left(\frac{\log t}{t}\right)^{12}
$$

Note that this formula agrees only partially with (3 18). Indeed, as we show, the coefficient has to depend on the initial data through $a^{\prime}(0)$ Furthermore, (1 15) implies that the second term of the phase is of order $\log ^{2} t$, while we find that it has to be $\log t$

The method of this paper follows the spirit of the work of Deift and Zhou (see [DZ], [DVZ]), who invented a new (and for the first time rigorous) method for recovering long-time asymptotics of integrable "soliton" equations, by using the fact that the inverse scattering problem for such equations can be stated as a Riemann-Hilbert factorization problem. We also make use of results of Deift, Its and Zhou for the defocusing nonlinear Schroedinger equation ([DIZ]). We note that the present work is the first in this spirit that deals with problems for which the jump matrix blows up at a point

\section{Away From a Singularity}

As mentioned in the introduction, Deift, Its and Zhou have analyzed the long time behavior of the defocusing nonlinear Schroedinger equation In that case, the Riemann-Hilbert problem agrees (modulo a minus sign) with (112)-(113) except that no spectral singularities exist at all (real or non-real) The question is how is the analysis of the problem affected when $\Psi$ and the jump matrix in (112) have a singularity In this section, we show that when for all singularities $\lambda^{\prime}, \lambda_{0}-\lambda^{\prime}=O(1)$, they have no effect at all We will only restrict ourselves to the case $\lambda_{0}>\lambda^{\prime}$, since obviously the case $\lambda_{0}<\lambda^{\prime}$ is similar (and easier) 
1 We begin by considering an auxiliary scalar factorization problem. Let $d$ be a function analytic in $\mathbb{C} \backslash\left(-\infty, i_{0}\right]$ such that

$$
\begin{aligned}
& d_{+}(\lambda)=d_{-}(\hat{\lambda})\left(1+|r(\hat{i})|^{2}\right) \quad \text { for }-\infty<\hat{\lambda}<\lambda_{0}, \\
& d_{+}(\hat{\lambda})=d_{-}(\hat{i}) \quad \text { for } \lambda>\lambda_{0} \\
& d \rightarrow 1 \quad \text { as } \lambda \rightarrow \infty
\end{aligned}
$$

Proposition 2.1. Problem (2 1) has a unique solution which has no zeros and whose only, singularities are at zeros of a More precisely, near a zero of $a, d_{+}(\lambda) a(i)$, $d(\lambda) a(\lambda), \frac{d-(\gamma)}{\bar{a}(/)}$ and $\frac{d(\lambda)}{a^{*}(\lambda)}$ are bounded above and below

Proof First note that

$$
1+|r(\hat{i})|^{2}=\frac{1}{|a(\lambda)|^{2}}=\frac{1}{a(\lambda) a^{*}(\lambda)}, \quad \text { for } i \in \mathbb{R}
$$

Also recall that $a$ is analytic in the upper half-plane, $a^{*}$ is analytic in the lower half-plane and $a(\lambda)=1+O\left(\frac{1}{i}\right)$ as $\lambda \rightarrow \infty, \operatorname{Im} \lambda \geqq 0$

Consider the contour depicted in Fig 21 (the choice of lines $l_{2}$ and $l_{3}$ are irrelevant provided $a, a^{*}$ have no zeros in regions $A_{2}, A_{3}$ or on lines $l_{2}, l_{3}$ ) and define

$$
\begin{aligned}
& \dot{\delta}(i)=d(i), \quad i \in A_{1}, \\
& \delta(i)=d(i) a(i), \quad i \in A_{2}, \\
& \delta(i)=d(i)\left(a^{*}(i)\right)^{-1}, \quad i \in A_{3} .
\end{aligned}
$$

$\delta$ satisfies the following scalar problem. it is analytic in $\mathbb{C} \backslash\left(l_{2} \cup l_{3}\right)$ and,

$$
\begin{aligned}
& \delta_{+}=\delta_{-} a, \quad \text { on } l_{2}, \\
& \delta_{+}=\delta_{-}\left(a^{*}\right)^{-1}, \quad \text { on } l_{3}
\end{aligned}
$$

As $a$ has neither zeros nor poles on $l_{2} \cup l_{3}$ we see that this scalar factorization problem has a unique solution with neither zeros nor poles, even though $a$ has a discontinuity at $\hat{i}_{0}, \delta$ is bounded near $\hat{i}_{0}$ (see e.g [G], p 448) The result follows

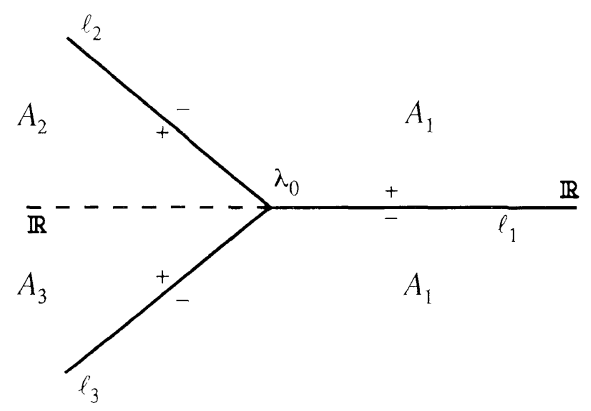

Fig. 2.1. 
2 We will next provide an appropriate contour deformation (following [DIZ]) which will be guided by an analysis of the signature of the phase $\Theta=i x+2 \lambda^{2} t$ appearing in the exponents of ( 112 ) (see Fig. 2 2)

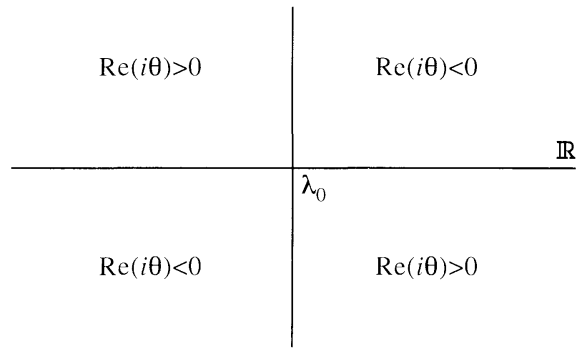

Fig. 2.2.

A fundamental fact is that the jump matrix of (1.12) admits the following factorizations (hence justifies our construction of $d$ )

$$
\left(\begin{array}{cc}
d & 0 \\
\bar{r} d e^{-2 \iota \Theta} & d^{-1}
\end{array}\right)\left(\begin{array}{cc}
d^{-1} & r d^{-1} e^{2 i \Theta} \\
0 & d
\end{array}\right) \text { for } \lambda>\lambda_{0}
$$

and

$$
\left(\begin{array}{cc}
d_{-} & \frac{i d_{-}^{-1} e^{2 \ell \Theta}}{1+|r|^{2}} \\
0 & d_{-}^{-1}
\end{array}\right)\left(\begin{array}{cc}
d_{+}^{-1} & 0 \\
\frac{\bar{r} d_{+} e^{-2 \ell}}{1+|r|^{2}} & d_{+}
\end{array}\right), \quad \text { for } \hat{\imath}_{\iota}<\hat{i}_{0}
$$

We deform our contour as follows (see Fig 23 )

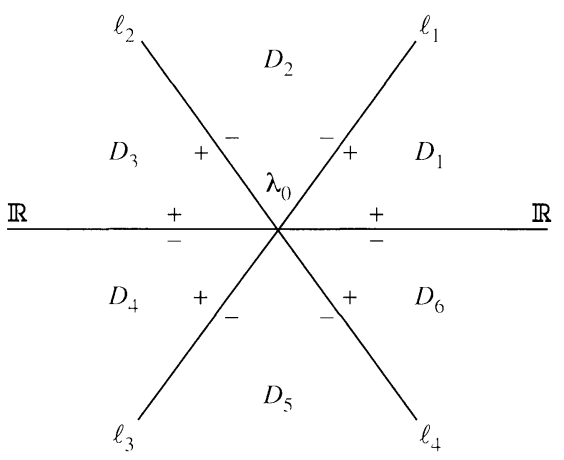

Fig. 2.3. 
Guided by the above factorizations, we define

$$
\begin{aligned}
& \Psi^{1}=\Psi\left(\begin{array}{cc}
d & -r d^{-1} e^{2 \ell \Theta} \\
0 & d^{-1}
\end{array}\right), \lambda \in D_{1}, \\
& \Psi^{1}=\Psi\left(\begin{array}{cc}
d & 0 \\
0 & d^{-1}
\end{array}\right), \quad \lambda \in D_{2}, \\
& \Psi^{1}=\Psi\left(\begin{array}{cc}
d & 0 \\
\frac{-\bar{r} d}{1+\left.i\right|^{2}} e^{-2 i \Theta} & d^{-1}
\end{array}\right), \quad i \in D_{3}, \\
& \Psi^{1}=\Psi\left(\begin{array}{cc}
d & \frac{\mid d^{-1} e^{2 \ell \theta}}{1+|r|^{2}} \\
0 & d^{-1}
\end{array}\right), \quad \lambda \in D_{4}, \\
& \Psi^{1}=\Psi\left(\begin{array}{cc}
d & 0 \\
0 & d^{-1}
\end{array}\right), \quad \lambda \in D_{5}, \\
& \Psi^{1}=\Psi\left(\begin{array}{cc}
d & 0 \\
\bar{r} d e^{-2 \iota \Theta} & d^{-1}
\end{array}\right), \quad i \in D_{6}
\end{aligned}
$$

Remark For such a deformation we need to assume that $b, \bar{b}$ can be analytically extended, at least in a small strip containing the real line (note that the actual choice of the curves $l_{j}$ is not important as long as they are in the right quadrant) This would be indeed true under more restrictive data ((110) for example). However, such an assumption is not necessary. As shown in [DZ] (see also [DIZ]) $b$ can be harmlessly approximated by a rational function whose poles do not affect the analysis

A straightforward calculation shows that there is no jump across the real line We have

$$
\Psi^{1}(\infty)=I
$$

The jump relation is

$$
\Psi_{+}^{1}=\Psi_{--}^{1} u_{\imath, t}^{1},
$$

where

$$
\begin{aligned}
u_{r, t}^{1} & =\left(\begin{array}{cc}
1 & -r d^{-2} e^{2 l \Theta} \\
0 & 1
\end{array}\right), \quad \text { on } l_{1}, \\
& =\left(\begin{array}{cc}
1 & 0 \\
\frac{-\bar{r} d^{2} e^{-2 \ell \Theta}}{1+|r|^{2}} & 1
\end{array}\right), \quad \text { on } l_{2}, \\
& =\left(\begin{array}{cc}
1 & \frac{\mid d^{-2} e^{2 \ell \Theta}}{1+|r|^{2}} \\
0 & 1
\end{array}\right), \quad \text { on } l_{3}, \\
& =\left(\begin{array}{cc}
1 & 0 \\
\bar{r} d^{2} e^{-2 l \Theta} & 1
\end{array}\right) \quad \text { on } l_{4} .
\end{aligned}
$$


3 The important observation is that this is a Riemann-Hilbert factorization problem without singularities at all. Indeed, the jump matrices have no poles as $a$ has no zeros on $l_{1} \cup l_{2} \cup l_{3} \cup l_{4}$ Also, $\Psi_{1}$ has no poles even at the points where $a(\lambda)=0$ This follows easily from the scattering relations (17), the definition of $\Psi$ (1 11), and Proposition 21 , which gives the behavior of $d_{ \pm}$near the zeros of $a$.

In other words, we end up with exactly the same problem as the one corresponding to initial data that produce no real spectral singularities at all Hence, the analysis of Deift, Its and Zhou goes through completely unaltered Note also that the solution of $(1.1)-\left(\begin{array}{ll}1 & 2\end{array}\right)$ is still recovered in the same way (see $\left.\left(\begin{array}{ll}1 & 14\end{array}\right)\right)$ since the modifications above have no effect on $\Psi$ up to order $\frac{1}{1}$

We will not provide the analysis of Deift, Its and Zhou in detail We refer the reader to [DIZ] instead We will only recall that as the jumps (24) are exponentially small away from the stationary phase point $\lambda_{0}$ the problem is reduced to one on a small cross near $\lambda_{0}$ The new problem can be solved explicitly (after some rescaling) in terms of parabolic functions For the reader's convenience we provide the leading order asymptotics for $q$

Theorem 2.1. Let $M>1, K>0$ be fixed and assume that for any real zero of a, say $\lambda^{\prime}$, we have $\lambda-\lambda^{\prime} \leqq K$ We have

$$
q(x, t)=t^{-12} \alpha\left(i_{0}\right) e^{\frac{i \mid x^{2}}{4 t}-w\left(i_{0}\right) \log (8 t)}+E(x, t),
$$

where, as $t \rightarrow \infty$,

$$
E(x, t)=O\left(t^{-1} \log t\right), \quad \text { for }\left|i_{0}\right| \leqq M,
$$

and for any' $j$,

$$
E(x, t)=O\left(|x|^{-1}+c_{j}\left(\lambda_{0}\right) x^{-1} \log |x|\right), \text { for }\left|\lambda_{0}\right| \geqq M^{-1},
$$

where

$$
\begin{gathered}
y(\lambda)=\frac{1}{2 \pi} \log \left(1+|r(\lambda)|^{2}\right)>0, \\
\left|\alpha\left(\lambda_{0}\right)\right|=\frac{v\left(\lambda_{0}\right)}{2}, \\
\arg x\left(\lambda_{0}\right)=\arg \Gamma\left(i v\left(\lambda_{0}\right)\right)-\arg r\left(\lambda_{0}\right)+\frac{\pi}{4}+\frac{1}{\pi} \int_{-\infty}^{i_{0}} \log \left(\lambda_{0}-\lambda_{0}\right) d\left(\log \left(1+\left|r\left(\lambda_{)}\right)\right|^{2}\right) .\right.
\end{gathered}
$$

\section{Near a Singularity}

In this section we consider the effect of a real spectral singularity which is close enough to the stationary phase point $\lambda_{0}$ For simplicity, we will assume that there are no non-real singularities and that there is only one real simple singularity, i.e. $a$ has a simple zero, say at zero (but see Sect 4 about these assumptions) We write $a(\lambda)=\lambda \tilde{a}(\hat{i})$, with $\tilde{a}(\hat{i}) \neq 0$

In this case, the analysis of [DIZ] breaks down, so the method of Sect 2 is no longer useful The behavior of $d$ (the solution of problem (21)) is more complicated near $\lambda_{0}$, it is no longer bounded there, and the relevant resolvent operators are also unbounded, so the standard method of [DIZ] cannot be applied directly. We will instead study this case by deforming the original problem in a different way 


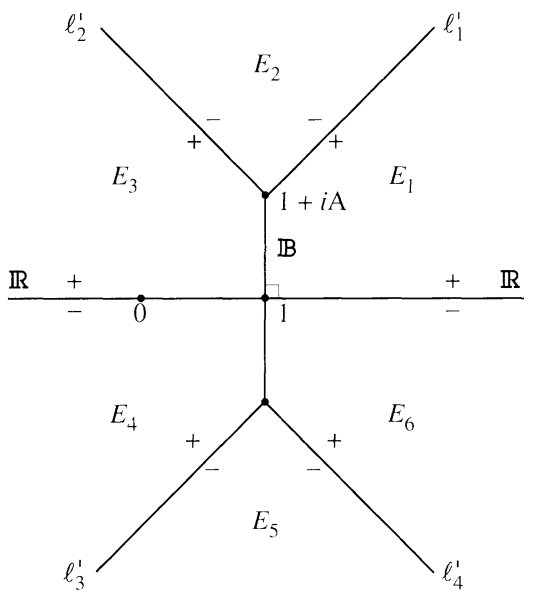

Fig. 3.1.

1 Let

$$
\tau=\frac{x^{2}}{16 t}=t i_{0}^{2}
$$

We consider the region defined by

$$
\lambda_{0} \rightarrow 0, \quad \tau \rightarrow \infty, \quad \text { as } t \rightarrow \infty
$$

Our first step will be to rescale appropriately so that the distance between the spectral singularity and the stationary phase point is $O(1)$. We next introduce a contour deformation (different from Sect. 2) which is still guided by the signature of the phase $\Theta$ It now turns out that, in the region we are interested in, the factorization problem takes a very special shape After a final conjugation involving an appropriate multi-valued function we end up with a problem on a vertical band, that can be solved in terms of the genus- 0 algebro-geometric solution of Eq. (1.1)

Remark A comprehensive reference for scalar problems with singular jumps (like $(21))$ is the book by Gakhov ([G])

2 Let $\Psi^{(1)}(\hat{i})=\Psi\left(\lambda_{0} \hat{\imath}\right)$ Condition (1 13) becomes

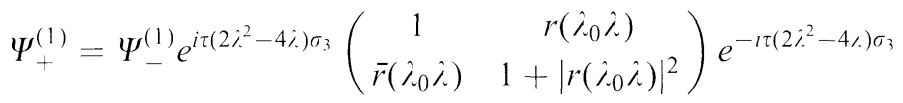

The rescaled phase is

$$
\Phi=\tau\left(\lambda^{2}-2 \hat{i}\right)
$$

The singularity of the problem is now at $\lambda=0$ while the stationary phase point is at $i=1$

3 Let $d$ be the solution of the scalar problem (21) We introduce a new contour $\Sigma$ as shown in Fig. 3 1. We denote the vertical band joining points $1+i A$ and $1-i A$ by $\mathbb{B} A$ is a real positive parameter depending on $\lambda_{0}$ and $\tau$, to be specified later 
Let

$$
\begin{aligned}
& \Psi^{(2)}=\Psi^{(1)}\left(\begin{array}{cc}
D & -R D^{-1} e^{2 l \Phi} \\
0 & D^{-1}
\end{array}\right), \hat{\lambda} \in E_{1} \\
& \Psi^{(2)}=\Psi^{(1)}\left(\begin{array}{cc}
D & 0 \\
0 & D^{-1}
\end{array}\right), \lambda \in E_{2}, \\
& \Psi^{(2)}=\Psi^{(1)}\left(\begin{array}{cc}
D & 0 \\
\frac{-\bar{R} D}{1+|R|^{2}} e^{-2 l} \Phi & D^{-1}
\end{array}\right), \lambda \in E_{3}, \\
& \Psi^{(2)}=\Psi^{(1)}\left(\begin{array}{cc}
D & \frac{R D^{-1} e^{2 / \phi}}{1+|R|^{2}} \\
0 & D^{-1}
\end{array}\right), \lambda \in E_{4}, \\
& \Psi^{(2)}=\Psi^{(1)}\left(\begin{array}{cc}
D & 0 \\
0 & D^{-1}
\end{array}\right), \lambda \in E_{5}, \\
& \Psi^{(2)}=\Psi^{(1)}\left(\begin{array}{cc}
D & 0 \\
\bar{R} D e^{-2 \imath \Phi} & D^{-1}
\end{array}\right), \lambda \in E_{6}
\end{aligned}
$$

Here $R(\hat{\lambda})=r\left(\lambda_{0} \lambda\right)$ and $D(\hat{i})=d\left(\lambda_{0} \lambda\right)$

A straightforward calculation shows that there is no jump across the real line We also have

$$
\Psi^{(2)}(\infty)=I
$$

The jump conditions for $\Psi^{(2)}$ are

$$
\Psi_{+}^{(2)}=\Psi_{-}^{(2)} u_{x, \tau}^{(2)}
$$

where

$$
\begin{aligned}
u_{\mathrm{r}, \tau}^{(2)} & =\left(\begin{array}{cc}
1 & -R D^{-2} e^{2 i \Phi} \\
0 & 1
\end{array}\right), \quad \text { on } l_{1}^{\prime}, \\
& =\left(\begin{array}{cc}
1 & 0 \\
\frac{-\bar{R} D^{2} e^{-2(\phi)}}{1+|R|^{2}} & 1
\end{array}\right), \quad \text { on } l_{2}^{\prime}, \\
& =\left(\begin{array}{cc}
1 & \frac{R D^{-2} e^{2 \ell \phi}}{1+|R|^{2}} \\
0 & 1
\end{array}\right), \quad \text { on } l_{3}^{\prime}, \\
& =\left(\begin{array}{cc}
1 & 0 \\
\bar{R} D^{2} e^{-2 i \Phi} & 1
\end{array}\right), \quad \text { on } l_{4}^{\prime},
\end{aligned}
$$




$$
\begin{aligned}
& =\left(\begin{array}{cc}
1 & -R D^{-2} e^{2 \imath \Phi} \\
\frac{\bar{R} D^{2} e^{-2 l}}{1+|R|^{2}} & \frac{1}{1+|R|^{2}}
\end{array}\right), \quad \text { on the top half of } \mathbb{B}, \\
& =\left(\begin{array}{cc}
\frac{1}{1+|R|^{2}} & \frac{-R D^{-2} e^{2 l \phi}}{1+|R|^{2}} \\
\bar{R} D^{2} e^{-2 i \Phi} & 1
\end{array}\right), \quad \text { on the bottom half of } \mathbb{B}
\end{aligned}
$$

Once more this is a Riemann-Hilbert factorization problem without singularities at all The proof of this is the same as in Sect. 2

4 We observe that, as in Sect 2, because of the structure sign of $\Phi$ the jumps across $\Sigma \backslash \mathbb{B}$ are exponentially close to $I$ as $\tau \rightarrow+\infty$ We thus end up with a Riemann-Hilbert factorization problem across $\mathbb{B}$.

Theorem 3.1. Let $\Psi^{(3)}$ be analytic in $\mathbb{C} \backslash \mathbb{B}$, such that

$$
\Psi_{+}^{(3)}=\Psi_{-}^{(3)} u_{x, \tau}^{(3)} \text { on }
$$

where

$$
\begin{aligned}
u_{\uparrow, \tau}^{(3)} & =\left(\begin{array}{cc}
1 & -R D^{-2} e^{2 l \Phi} \\
\frac{\bar{R} D^{2} e^{-2 i \Phi}}{1+|R|^{2}} & \frac{1}{1+|R|^{2}}
\end{array}\right), \quad \text { on the top half of } \mathbb{B}, \\
& =\left(\begin{array}{cc}
\frac{1}{1+|R|^{2}} & \frac{-R D^{-2} e^{2 i \phi}}{1+|R|^{2}} \\
\bar{R} D^{2} e^{-2 i \Phi} & 1
\end{array}\right), \quad \text { on the bottom half of } \mathbb{B} .
\end{aligned}
$$

and

$$
\Psi^{(3)}(\infty)=I .
$$

Then $\Psi^{(2)}-\Psi^{(3)}=O\left(\tau^{-l}\right)$, for any positive $l$, as $\tau \rightarrow \infty$, uniformly in $x$, in compact subsets of the i-Riemann-sphere

Proof The details of the proof are omitted (see [DZ]) The important observation here is that $R D^{-2}$ and $\frac{\vec{R} D^{2}}{1+|R|^{2}}$ are actually under control on the top half of $\mathbb{B}$ while $\frac{-R D^{-2}}{1+|R|^{2}}$ and $\bar{R} D^{2}$ are under control on the bottom half For example (again see [G], p 448) $D$ behaves like $\lambda_{0}^{-1 / 2}(\lambda-1)^{-1 / 2}$ and $R D^{-2}$ is bounded Note here that it is crucial to chose the segment $\mathbb{B}$ at right angles with the real line; the behavior of $D$ depends essentially on the direction in which we approach $\lambda=1$.

5 We introduce the multi-valued function

$$
\Omega=2(\lambda-1)\left((\lambda-1)^{2}+A^{2}\right)^{1 / 2}-A^{2}-2 .
$$

We consider this as a function on $\mathbb{C}$, chosing the branch consistently with the condition

$$
\Omega=2 \lambda^{2}-4 \lambda+O\left(\frac{1}{\lambda^{2}}\right), \text { as } \lambda \rightarrow \infty
$$


Naturally, $\Omega$ has a jump across $\mathrm{B}$ Also,

$$
\begin{aligned}
\Omega_{+}+\Omega_{-} & =-2\left(A^{2}+2\right), \\
i\left(\Omega_{+}-\Omega_{-}\right) & <0, \quad \text { on the top half of } \mathbb{B}, \\
i\left(\Omega_{+}-\Omega_{-}\right) & >0, \quad \text { on the bottom half of } \mathbb{B}
\end{aligned}
$$

Defining

$$
\Psi^{(4)}=\Psi^{(3)} e^{\imath(2 \Phi-\tau \Omega)},
$$

we end up with the following Riemann-Hilbert problem.

$$
\Psi_{+}^{(4)}=\Psi_{-}^{(4)} u_{r, \tau}^{(4)},
$$

where

$$
\begin{gathered}
u_{\uparrow, \tau}^{(4)}=\left(\begin{array}{cc}
e^{i \tau\left(\Omega_{+}-\Omega_{-}\right)} & -R D^{-2} e^{i \tau\left(\Omega_{+}+\Omega_{-}\right)} \\
\frac{\bar{R} D^{2} e^{-\tau \tau\left(\Omega_{+}+\Omega_{-}\right)}}{1+|R|^{2}} & \frac{1}{1+|R|^{2}} e^{-i \tau\left(\Omega_{+}-\Omega_{-}\right)}
\end{array}\right), \quad \text { on the top half of } \mathbb{B}, \\
=\left(\begin{array}{cc}
\frac{e^{i \tau\left(\Omega_{-}-\Omega_{-}\right)}}{1+|R|^{2}} & \frac{-R D^{-2} e^{\tau \tau\left(\Omega_{+}+\Omega_{-}\right)}}{1+|R|^{2}} \\
\bar{R} D^{2} e^{-l \tau\left(\Omega_{+}+\Omega_{-}\right)} & e^{-l \tau\left(\Omega_{+}-\Omega_{-}\right)}
\end{array}\right), \quad \text { on the bottom half of } \mathbb{B}, \\
\Psi^{(4)}(\infty)=I
\end{gathered}
$$

Remark The choice of $\Omega$ is inspired by the theory of algebro-geometric solutions of the NLS equation (cf [BBEIM]) We are seeking a generalized differential $\tau d \Omega$ on a Riemann surface, whose integral behaves like the phase $2 \Phi$ as $\lambda \rightarrow \infty$ The actual Riemann surface (of genus 0 in our case) is dictated by the Riemann-Hilbert contour $\mathbb{B}$ in $\left(\begin{array}{ll}3 & 8\end{array}\right)$

6 As it stands, (3 14) does not look like an improvement over the original RiemannHilbert problem. However, we shall now show that near the "shock" region, it can be much simplified. In this subsection, we consider the "shock front," i e the region II of Theorem 1.1

We choose A such that $\left|\lambda_{0}\right|=e^{-\tau A^{2}}$, i e

$$
A=\left(\frac{-\log \left|\lambda_{0}\right|}{\tau}\right)^{1,2}
$$

$A$ and $\tau$ depend on $x$ and $t$ and should be thought of as a "slow" and a "fast" variable, respectively 
Because of (3 15), the diagonal terms of $u_{r, \tau}^{(4)}$ tend rapidly to 0 , as $\tau \rightarrow \infty$ As in Subsect. 4, we end up with

$$
\Psi_{+}^{(5)}=\Psi_{-}^{(5)} u_{r, \tau}^{(5)}
$$

where

$$
\begin{gathered}
u_{r, \tau}^{(5)}=\left(\begin{array}{cc}
0 & C e^{-\imath \tau\left(2 A^{2}+4\right)} \\
-\frac{1}{C} e^{i \tau\left(2 A^{2}+4\right)} & 0
\end{array}\right), \lambda \in \mathbb{B}, \\
\Psi^{(5)}(\infty)=I,
\end{gathered}
$$

where

$$
C=-\frac{b(0)}{a^{\prime}(0)} \exp \left(\frac{1}{2 \pi i} \int_{-\infty}^{0} \log (-s) d \log \left(|\tilde{a}(s)|^{2}\right)\right)
$$

Note that there is no discontinuity at $\lambda=1$. Here one uses the fact that when $a(\lambda)=0,|b(\lambda)|=1($ see $(18))$, hence $\bar{b}(0)=\frac{1}{b(0)}$.

Problem (3 16) can be solved explicitly It can be interpreted as a form of the Riemann-Hilbert problem for the genus-0 algebro-geometric solution of (1 1) (see e g. [MA], [LM], [BBEIM, ch 4]. We analyze and solve $(316)$ in the appendix

Keeping track of the different transformations of $\Psi$ and recalling (1 14), we have.

Theorem 3.2. The effect of a real spectral singularity at $\lambda^{\prime}$ is only felt in the region $\lambda^{\prime}-\lambda_{0} \rightarrow 0$ In the case, say, $\lambda^{\prime}=0$ we have, as $\lambda_{0} \rightarrow 0$ and $\tau \rightarrow \infty$,

$$
\begin{aligned}
q(x, t) \sim & \frac{\left(-\log \left(\left|\lambda_{0}\right|\right)\right)^{1 / 2}}{\left|a^{\prime}(0)\right| t^{1 / 2}} \cdot \exp \left(-4 i \tau+2 i \log \left(\left|\lambda_{0}\right|\right)+i \arg (b(0))\right. \\
& \left.-i \arg a^{\prime}(0)-\frac{i}{2 \pi} \int_{-\infty}^{0} \log (-s) d \log |\tilde{a}(s)|^{2}\right),
\end{aligned}
$$

where $\lambda_{0}=\frac{-x}{4 t}, a, b$ are the scattering coefficients defined in $(1.7)$ and $\tilde{a}(s)=\frac{a(s)}{s}$

In particular, in the region $\frac{-x}{4 t} \sim\left(\frac{\log t}{t}\right)^{1 / 2}$ the amplitude of the solution decays like $O\left(\left(\frac{\log t}{t}\right)^{1 / 2}\right)$.

7 The region $\tau \rightarrow \infty, \lambda_{0} \rightarrow 0$ corresponds to the "front" of the region in which the effect of the real singularity is felt We conclude this section by considering the region where $\tau$ is bounded and $\hat{\lambda}_{0} \rightarrow 0, t \rightarrow \infty$

We now use a different rescaling, i e $\lambda \rightarrow i \frac{(\log t)^{12}}{t^{12}}$. The phase becomes

$$
\Theta=2 \lambda^{2} \log t+\frac{x}{t^{1 / 2}}(\log t)^{1 / 2} \lambda .
$$

The function $\Omega$ (see (3.10)) should now be

$$
\Omega=2 \log t(\lambda+1)\left(\lambda-1+\left((\lambda-1)^{2}+A^{2}\right)^{1 / 2}\right)+\frac{x}{t^{1 / 2}}\left(\lambda+\left((\lambda-1)^{2}+A^{2}\right)^{1 / 2}\right)
$$

We still define $A$ by (3.15) and we still end up with a Riemann-Hilbert problem on a vertical band. Following the steps outlined in Sect. 6, we end up with the following asymptotics for $q$. 
Theorem 3.3. In region III, with $\lambda_{0} \rightarrow 0$, $\tau$ bounded, as $t \rightarrow \infty$, the asymptotics for the solution of $(11),(1.2)$ is

$$
q(x, t) \sim C t^{-1 / 2} \frac{\hat{\lambda}_{0} \log \left|\hat{\lambda}_{0}\right|}{\tau} \exp \left(-2 i\left(A^{2}+2\right) \log t+\frac{2 i x}{t^{1 / 2}}(\log t)^{1 / 2}\right),
$$

where $\lambda_{0}, \tau, A$ are as above, and $C$ is defined by (3 17)

\section{Higher Order Zeros and Finitely Many Singularities}

It should be clear by the discussion of Sects 2 and 3 that there is nothing special about a singularity at zero In the general case of finitely many singularities the following is true. for each real singularity $\lambda_{l}$, there is a region where $\lambda_{i}-\lambda_{0} \rightarrow 0$, as $t \rightarrow \infty$, in which the solution decays with leading asymptotics similar to (3 18) and (3.19) Non-real singularities correspond to solitons as usual (see e g [FT], $[\mathrm{K}]$ for the infinite case).

On the other hand, it is clear from Sect. 3 that the order of the zero of $a$ is not important, the order of the solution remains the same but there is a minor change in the phase We leave the details to the reader

As mentioned in Sect 1, a study of the interesting generalization of our result in the case of infinitely many real singularities is under way (for examples of such initial data see $[Z])$.

\section{Appendix. The Riemann-Hilbert Problem for the Genus-0 Solution}

In this appendix we solve the Riemann-Hilbert problem (3 16) We do this by diagonalizing the jump matrix and thus reducing the problem to a scalar one which can then be solved explicitly.

Let $G=C \exp \left(-i \tau\left(2 A^{2}+4\right)\right)$ and

$$
S=\left(\begin{array}{cc}
-i G & 1 \\
1 & -\frac{i}{G}
\end{array}\right)
$$

The eigenvalues of $S$ are $i$ and $-i$, we have

$$
\left(\begin{array}{cc}
0 & G \\
-\frac{1}{G} & 0
\end{array}\right)=S\left(\begin{array}{cc}
i & 0 \\
0 & -i
\end{array}\right) S^{-1} .
$$

Define

$$
\Lambda=\left(\frac{\hat{i}-1+i A}{i-1-i A}\right)^{14} .
$$

Then $\Lambda$ solves the scalar problem

$$
\begin{gathered}
\Lambda_{+}=\Lambda_{-} i, \text { on } \mathbb{B}, \\
\Lambda(\infty)=1
\end{gathered}
$$


Let the matrix $\mu$ be defined by

$$
\mu=S\left(\begin{array}{cc}
\Lambda & 0 \\
0 & \Lambda^{-1}
\end{array}\right) S^{-1}
$$

Then

$$
\begin{gathered}
\mu_{+}=\mu_{-}\left(\begin{array}{cc}
0 & G \\
-\frac{1}{G} & 0
\end{array}\right), \text { on } \mathbb{B}, \\
\mu(\infty)=I
\end{gathered}
$$

Thus $\mu$ is the solution of (3.16) Furthermore, near infinity,

$$
\mu(\hat{i})=I+\left(\begin{array}{cc}
0 & -\frac{G A}{2(-1-1 A)} \\
-\frac{2}{G A(i-1-i A)} & 0
\end{array}\right)
$$

In particular $\lim _{i \rightarrow \infty}\left(2 \lambda \mu_{12}\right)=-G A$ The asymptotics of $q$ is now immediately recovered through (1 14$)$.

Remark One can interpret problem (3.16) as the Riemann-Hilbert problem for the genus-0 solution of the NLS equation Although the use of the term "algebrogeometric solution" may sound pretentious in the very simple special case of genus 0 , it is worth pointing out the fact that what we encounter here is an instance of a very general phenomenon, where the long-time behavior of the solution of a soliton equation in a particular region is related to a different type of solution, which is indeed connected with Riemann surfaces, and the associated theory of theta functions connected with the Abel map For a discussion of that theory in the context of the nonlinear Schroedinger equation (focusing and defocusing) see [MA], [LM] and [BBEIM, ch 4]

Acknowledgements We wish to thank Jean-Claude Guillot for pointing our attention to this problem and for references $[\mathrm{CK}]$ and $[\mathrm{F}]$ and illuminating discussions We also thank Percy Deift for discussions concernmng Riemann-Hilbert problems on a Riemann surface, Daniel Battig for discussions concerning the periodic problem for the NLS system and Dan Dix for helpful discussions and for pointing out reference [AS] Finally we thank the referees for helpful comments concerning our presentation

\section{References}

[AS] Ablowitz, M J, Segur, H : Asymptotic Solutions of the Korteweg-de Vries Equation Studies in Applied Mathematics 57, n 1, 13-44 (1977)

[BBEIM] Beiokolos, VD, Bobenko, A I, Enolskii, VZ, Its, A R, Matveev, V Z: AlgebroGeometı1c Approach to Nonlinear Evolution Equations Berlin-Heidelberg-New York: Springet-Verlag, 1993

[BC] Beals, R, Coifman, R: Scattering and Inverse Scattering for Fist Ordet Systems Commun Pure and Appl Math 37, 39-90 (1984)

[CK] Cohen, A, Kappeler, T: Solutions to the Cubic Schroedinger Equation by the Inverse Scattering Method SIAM J Math Anal 23, n 4, 900--922 (July 1992)

[DIZ] Deift, P, Its, A R, Zhou, X : Important Developments in Soliton Theory 1980-1990, ed by A Fokas and VE Zakharov, Berlin-Heidelberg-New York: Springer-Verlag, 1993

[DVZ] Deift, P, Venakides, S, Zhou, X: The Collisionless Shock Region for the LongTime Behavior of Solutions of the KdV equation Commun Pure and Appl Math 47, 199-206 (February 1994) 
[DZ] Deift, P, Zhou, X : A Steepest Descent Method for Oscillatory Riemann--Hilbert Problems Annals Math (March 1993)

[DZ2] Deift, P, Zhou, X: Direct and Inverse Scattering on the Line with Arbitrary Singularities Commun Pure and Appl Math 44, 485-533 (1991)

[F] Folland, G : J Differ Eq 39, 151-185 (1981)

[FT] Fadeev, L D , Takhtajan, L A : Hamiltonian Methods in the Theory of Solitons BerlinHeidelberg-New York: Springer-Verlag, 1987

[G] Gakhov, F D : Boundary Value Problems London: Pergamon Press, 1966

[GKZ] Gesztesy, F, Karwowski, W, Zhao, Z : Duke Math J 68, 101-150 (1992)

[K] Kamvissis, S: Focusing NLS Equation with Infinitely Many Solitons J Math Phys (August 1995)

[LM] Li, Y, McLaughlin, D W : Morse and Melnikov Functions for NLS Pde's Commun Math Phys 162, 175-214 (1994)

[MA] Ma, Y C , Ablowitz, M J : The Periodic Cubic Schroedinger Equation Studies in Applied Mathematics 65, 113-158 (1981)

[NMPZ] Novikov, S, Manakov, S V, Pitaevskii, L P, Zakharov, V E : Theory of Solitons Consultants Bureau, New York: Plenum Publishing Company, 1984

[Z] Zhou, X : Direct and Inverse Scattering Transforms with Arbitrary Spectral Dependence Commun Pure and Appl Math 42, 895-938 (1989)

Communicated by J L Lebowitz 
